\title{
MODULATION OF THE ISFET RESPONSE BY AN IMMUNOLOGICAL REACTION
}

\author{
R. B. M. SCHASFOORT ${ }^{\dagger *}$, P. BERGVELD*, J. BOMER*, R. P. H. KOOYMAN ${ }^{\dagger}$ and \\ J. GREVE⿱ \\ Departments of Applied Physics ${ }^{\dagger}$ and Electrical Engineering*, Twente University of \\ Technology, P. O. Box 217, 7500 AE Enschede (The Netherlands)
}

\begin{abstract}
A new method is presented for the detection of an immunological reaction in a membrane. Antigens (or antibodies) are taken up in a porous membrane, which covers the gate area of an ISFET. By stepwise changing of the electrolyte concentration, a transient transport of ions through the membrane-protein layer occurs, resulting in a transient membrane potential, which is measured with the ISFET. The transport is determined by the fixed charge density in the protein layer, which changes upon formation of antibody-antigen complexes. No membrane potential is induced at zero fixed charge density. Isoelectric points can therefore be deduced from the registration, and this forms the basis for sensing an immunological reaction. We discuss this new measuring technique and show that antibody concentrations of $10^{-6} \mathrm{M}$ can easily be detected.
\end{abstract}

\section{Introduction}

Several research groups have tried to detect directly an immunological reaction by means of an immuno-modified ISFET. The detection of the charge redistribution caused by an immunological reaction has failed so far, because the protein charge cannot be detected in practice due to screening of the charges by small inorganic ions present in the sample [1]. From a.c. measurements we found that the specific conductivity in an immune precipitate, deposited on the ISFET surface, is even higher than in the surrounding buffer [2]. Here we introduce an alternative detection method for sensing an immunological reaction occurring in a membrane deposited on an ISFET, based on the measurement of a transient membrane potential as the result of a chemical disturbance of an equilibrium condition.

Materials and methods

The ISFETs used in the experiments were n-channel ISFETs with a $700 \AA$ thermally-grown $\mathrm{SiO}_{2}$ and $1200 \AA \mathrm{Ta}_{2} \mathrm{O}_{5}$ meander gate. We used $\mathrm{KCl}$ 
as the 'high salt' electrolyte, which had a conductivity of 5.0 times the buffer conductivity. The conductivity was measured with a Konsort K 820 conductivity meter. The buffers used where citric acid/KOH, $\mathrm{pH}=3.0$, $100 \mu \mathrm{S}$; acetic acid/KOH, $\mathrm{pH}=5.0,100 \mu \mathrm{S}$ and morpholino ethan sulfonic acid (Merck) (MES) $/ \mathrm{KOH}, \mathrm{pH}=6.0,100 \mu \mathrm{S}$. Human serum albumin (HSA) was purchased from Sigma, and anti human serum albumin (aHSA) was from Dakopath Denmark.

For the preparation of the protein support, we used polystyrene latex $2.5 \%, 0.12 \mu \mathrm{m}$ (Polyscience), which was mixed with $0.25 \%$ agarose (Biorad) type low IEE, zero Mr. This mixture was cast on a wafer containing the ISFETs with lift-off polyimide and cooled in a refrigerator at $4{ }^{\circ} \mathrm{C}$, resulting in a slow evaporation of the solvent leaving a porous membrane with a thickness of about $5 \mu \mathrm{m}$. After a temperature step to $55{ }^{\circ} \mathrm{C}$, the polyimide was removed and the membrane lifted off the undesired places on the wafer. In this way ISFETs with a latex membrane on top of the gate area were manufactured. Proteins could be absorbed to the support by incubation for a certain time.

The ISFETs were mounted on a piece of printed circuit board and encapsulated with $\mathrm{Hy}-\mathrm{Sol}$ epoxy, which was flattened for leak-free mounting in a flow-through system (see Fig. 1).

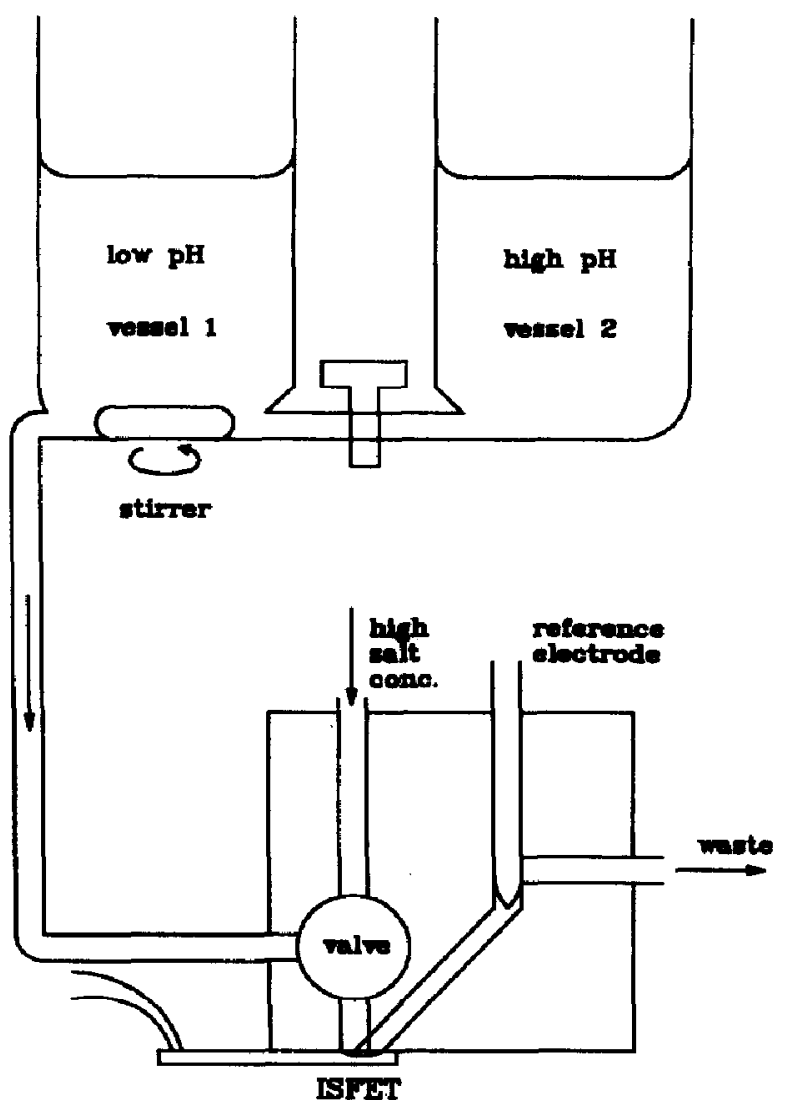

Fig. 1. Schematic presentation of the flow-through system. 
The $\mathrm{pH}$ of the equilibrium buffer could be changed by using a gradient vessel. When the solution flowed under hydrodynamic pressure out of vessel 1 , the $\mathrm{pH}$ changed through mixing with a solution of different $\mathrm{pH}$ from vessel 2. By opening the valve for a few seconds, the ISFET was exposed to a higher electrolyte concentration. The step change occurs within $50 \mathrm{~ms}$. After this step, the valve was closed and the membrane was again equilibrated with the buffer flowing out of vessel 1 . We connected the ISFETs to a source drain follower as reported elsewhere [3]. The responses were measured with an $X Y T$ recorder (Kipp and $\mathrm{Zn}, \mathrm{BD91}$ ).

Results

Upon increasing the electrolyte concentration by opening the valve, ions diffused into the protein layer adsorbed on the latex membrane caused by the induced concentration gradient. The diffusion of positive and negative ions was influenced differently by the fixed charge density in the protein-containing membrane. The different mobilities of cations and anions result in a transient membrane potential, which is measured with the ISFET. Upon closing the valve, the ensuing decrease of electrolyte concentration caused a reverse membrane potential (see Fig. 2).

We tested several membrane materials and found that a matrix made of polystyrene latex crosslinked with agarose was a suitable protein support.

If the $\mathrm{pH}$ of the buffer was changed, the fixed charge density was also changed due to the association-dissociation constants of the amino and carboxyl groups in the protein complex. Therefore the amplitude of the measured membrane potentials was influenced by the pH. In Fig. 3 typical responses are shown, which result from repeated increases in electrolyte concentration during a gradual $\mathrm{pH}$ change in time.

The response of the ISFET was an S-shaped curve, on which transients are superimposed. The S-shaped curve resulted from the gradual change in $\mathrm{pH}$ at constant ionic strength. The ISFET then functioned as a $\mathrm{pH}$ meter.

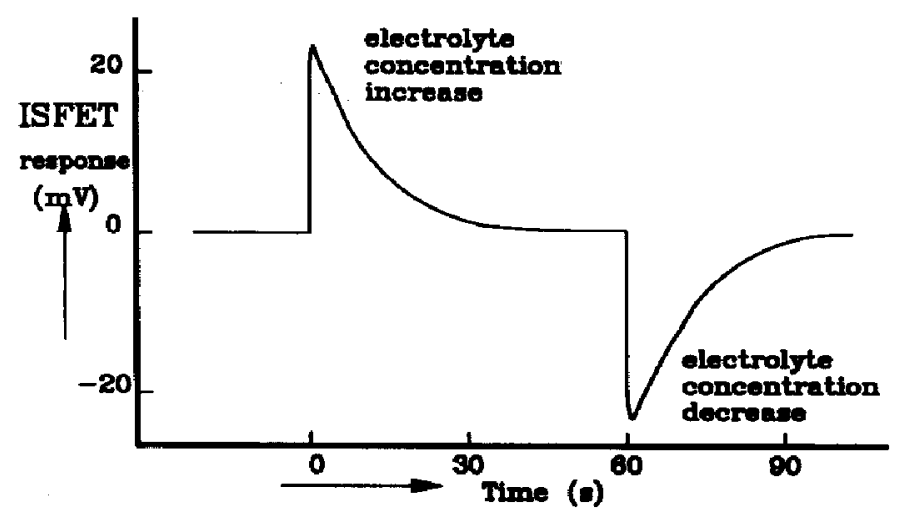

Fig. 2. Typical response of an ISFET with protein membrane to a stepwise change of electrolyte concentration. Electrolyte conductivity ratio of $5.0, \mathrm{pH}=\mathbf{7 . 0}$. 


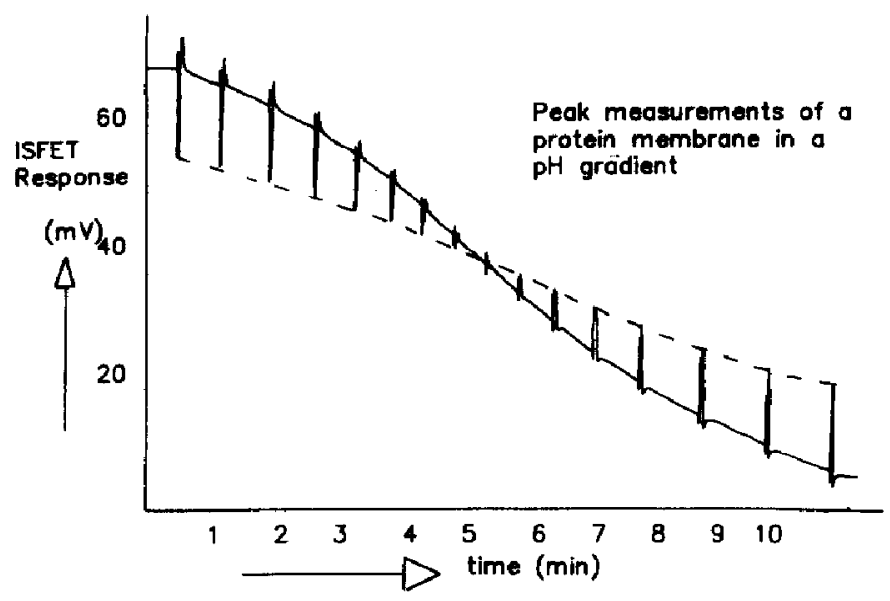

Fig. 3. S-shaped curve caused by the pH gradient, on which transients are superimposed due to a repeated stepwise increase of electrolyte concentration.

The transients were caused by the stepwise increases in salt concentration. At a certain $\mathrm{pH}$ no response was observed at all. This $\mathrm{pH}$ is called the inversion point ( $\left.\mathrm{pI}^{\prime}\right)$ of the protein complex for the specific measuring condition.

An immunological reaction was determined in the following way. First with a non-protein loaded membrane a 'bias' membrane potential was measured as shown in Fig. 4 (line a). Then the membrane was incubated with $10^{-5} \mathrm{M}$ HSA for three hours, resulting in a change of inversion point ( $\left.\mathrm{pI}^{\prime}\right)$ and slope $\left(\delta \phi_{\mathrm{m}} / \delta \mathrm{pH}\right)$ (line b). As a control, the membrane was then

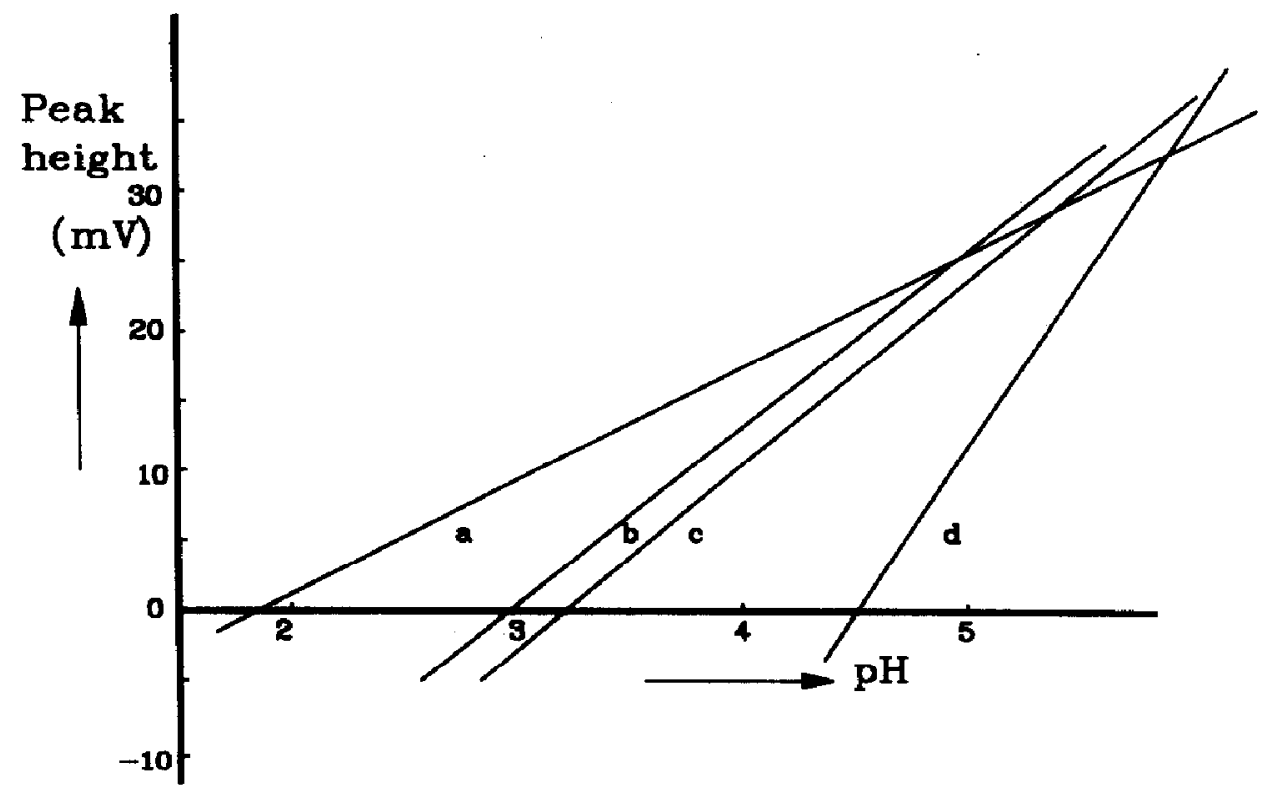

Fig. 4. Amplitude of the transient membrane potential as a function of $\mathrm{pH}$ : (a), bare polystyrene latex agarose membrane; (b), (a) plus adeorbed HSA, $10^{-6} \mathrm{M}, 3 \mathrm{~h}$; (c), (b) plus adsorbed aHCG, $10^{-5} \mathrm{M}, 1 \mathrm{~h}$; (d), (c) plus adsorbed aHSA, $10^{-6} \mathrm{M}, 1 \mathrm{~h}$. 
incubated with $10^{-5} \mathrm{M}$ aHCG (anti human chorion gonadotrophine) and only a small shift was observed due to aspecific adsorption to the matrix (line $\mathrm{c}$ ). Then the membrane was incubated with $10^{-6} \mathrm{M}$ aHSA for one hour. We found a rather strong shift of the $\mathrm{pI}^{\prime}$ point, as well as the slope $\delta \phi_{\mathrm{m}} / \delta \mathrm{pH}$, caused by the immunological reaction (line $\mathrm{d}$ ).

\section{Discussion and conclusions}

The net charge density in a protein layer deposited on an ISFET can be measured by exposing the membrane to a stepwise change in electrolyte concentration as indicated above. The transient membrane potential observed is a result of the different mobilities of the positive and negative ions in the protein layer. We observed characteristic inversion points and slopes $\left(\delta \phi_{\mathrm{m}} / \delta \mathrm{pH}\right)$ as a function of the protein concentration and type of protein. Also isoelectric points could be deduced from the membrane potentials as a function of the $\mathrm{pH}$.

This detection of the isoelectric point of a protein complex is the basis for the development of an immunological sensor. An immunological reaction results in a change of the fixed charge density in the membrane, which can be explained by a shift of the protein isoelectric point due to the immunological reaction.

We suppose that the amplitude of the measured membrane potentials corresponds to the weighted average of the fixed charge density in the protein complex. Theoretical aspects will be investigated in the future.

\section{Acknowledgement}

This investigation is part of the research program of the Stichting Fundamenteel Onderzoek der Materie (FOM), which is financially supported by the Nederlandse Organisatie voor Wetenschappelijk Onderzoek (NWO).

\section{References}

1 J. Janata, Chemical selectivity of field effect transitors, Proc. 2 nd Int. Meet. Chem. Sensors, Bordeaux, France, July 7 - 10, 1986, pp. 25 - 31.

2 R. B. M. Schasfoort, G. Streekstra, R. P. H. Kooyman, P. Bergveld and J. Greve, Influence of an immunological precipitate on d.c. and a.c. behaviour of an ISFET, submitted for publication in Sensors and Actuators.

3 P. Bergveld, The operation of an ISFET as an electronic device, Sensors and Actuators, 1 (1981) $17-29$. 